\title{
Procedimientos de Soldadura y Calificación de Soldadores: una Propuesta de Enseñanza y Guía de Aplicación para la Industria
}

\author{
Enrique E. Niebles y William G. Arnedo \\ Universidad Autónoma del Caribe, Facultad de Ingeniería, Programa de Ingeniería Mecánica, \\ Calle 90 No 46-112, Barranquilla-Colombia (e-mail: eniebles@uac.edu.co; eenniebles@yahoo.com)
}

\begin{abstract}
Resumen
Se presenta una propuesta de enseñanza de la soldadura que incluye modelo pedagógico aplicado, metodología de desarrollo y una guía para la elaboración de proyectos de aula, dirigido a estudiantes de Ingeniería Mecánica e Industrial, dentro del contexto de la formación por competencias. Se muestra un proyecto desarrollado en una empresa del sector de la soldadura, dirigido al control y aseguramiento de la unión soldada, usando la metodología propuesta. La aplicación del modelo motivó significativamente a los estudiantes en el estudio de la soldadura y procesos afines, vinculó a las empresas del sector con la universidad, generó documentos de trabajo desarrollado por estudiantes con asesorías de docentes y expertos de las empresas.
\end{abstract}

\section{Welding Procedures and Welder Qualifications: A Proposal for Teaching and Application Guide for the Industry}

\begin{abstract}
This paper presents a proposal for the teaching of welding processes that includes an applied pedagogical model, a development methodology and a guide for the preparation of classroom projects directed to students of Mechanical and Industrial Engineering, all under the concept of training by competency. A project developed in a company of the welding sector, directed to the control and quality of welded joints was done, using the proposed methodology. The application of the model significantly motivated the students in the learning of welding and related processes enhanced the relation with the industry, and generated important documents developed by students with advice of professors and professionals of the companies.
\end{abstract}

Keywords: welding teaching, training by competency, quality control, university-industry relation 


\section{INTRODUCCIÓN}

Se vive en un mundo internacionalizado, de globalización de la economía y de libre comercio, donde existe una elevada competencia desde el exterior, y la necesidad de encontrar nuevos mercados en otros países. Los códigos, especificaciones y procedimientos de soldadura son recomendados para su aplicación en la industrias metalmecánicas afines con la tecnología de soldadura dado que garantizan confiabilidad y aseguramiento en un producto terminado con óptima calidad debido a sus exigencias tecnológicas y son la documentación básica que rige y guía la práctica de soldadura aplicables para: i) fabricar productos soldados que cumplan con la calidad y seguridad del trabajador requerida, ii) suministrar una real y razonable protección a la vida, la propiedad y el medio ambiente. (Niebles et al., 2008)

En la actualidad, uno de los métodos más importantes para la unión de piezas y conjuntos metálicos en general en las industrias que aportan al producto interno bruto y adelanto en infraestructura de países desarrollados y en vías de desarrollo es la soldadura y sus tecnologías (Camillero et al., 2007; Wang y Liu, 2004; Méndez, 1999); por tanto es responsabilidad de las empresas fabricantes de productos soldados comprobar que la calidad de sus productos este de conformidad con los requerimientos del cliente y lo establecido en códigos y especificaciones de soldadura, y un primer paso para asegurar la calidad de sus productos y la idoneidad y habilidad de sus soldadores es elaborando procedimientos de soldadura que permitan garantizar la compatibilidad del metal de soldadura depositado con el metal base utilizado y calificando a sus soldadores y punteadores acorde con el procedimiento de soldadura calificado, esta evaluación de los soldadores es muy importante para la empresa, dado que permite garantizar el desarrollo de uniones soldadas de alta calidad y además da garantía, buen nombre y confiabilidad a la empresa como al soldador, responsables de la aplicación de soldadura. Así mismo es importante que dentro del contexto de investigación, innovación y desarrollo de procesos mixtos e híbridos en soldadura, de nuevos materiales base y de aporte, y caracterización mecánica y microestructural de soldaduras y recubrimientos superficiales llevadas a cabo por instituciones tecnológicas, universidades y centros de investigación; las aplicaciones de soldadura (Bolaños et al., 2006; Dunder, 2006; Baldomir et al., 2005) estén soportadas además de personal capacitado, calificado y entrenado para tal fin debe estar soportado también por procedimientos de fabricación, ensamble y soldadura que garanticen la confiabilidad de los resultados obtenidos y por ende de las uniones soldadas.

Sin embargo, existen antecedentes (Niebles, 2007; SENA, 2006) que demuestran que en las empresas del sector metalmecánico afines con la soldadura existe desconocimiento y poca evaluación e investigación de las tecnologías de soldadura, ingenieros con poco conocimiento del área de la soldadura que ocupen los cargos de supervisor e inspectores de control de calidad en esta área, y metodologías que faciliten el desarrollo y aseguramiento de calidad de sus productos soldados por tanto es responsabilidad de la instituciones de nivel tecnológico y superior en las áreas de ingeniería afines con la tecnología de soldadura enseñarlas, facilitando la integración de la teoría y la práctica de la soldadura en el contexto integrador Universidad - Empresa que potencialice en el estudiante la autonomía, la creatividad y la construcción de su propio conocimiento en esta área.

De aquí que el presente trabajo expone una propuesta de enseñanza dentro del contexto de formación por competencias de las tecnologías de soldadura que integra al estudiante de la universidad con la industria tomando como base el aprendizaje por proyectos y estrategias conexas, y a la vez presenta una metodología para la elaboración y calificación de procedimientos de soldadura y calificación de soldadores desde la perspectiva del control y aseguramiento de calidad resultado del proceso mismo de enseñanza en el aula.

\section{MODELO}

El modelo pedagógico utilizado por la universidad dentro de su proceso de formación por competencias es el modelo pedagógico integrado integrador que busca la formación integral y en valores del estudiante, mediante un proceso autorregulado y participativo en el contexto de la profesionalización e investigación que generen ciencia, innovación y desarrollo tomando como protagonistas activos a estudiantes, docentes, sociedad e industria. 
Para la planificación de actividades de formación dentro de este contexto de formación es importante establecer como, donde, cuando y con qué medios se va a formar las competencias, establecer los contenidos curriculares los cuales deben consignar además del problema profesional a resolver, la competencia a formar, la planeación del docente tomando en cuenta el numero de créditos y la distribución de las actividades de enseñanza - aprendizaje para el cumplimiento de los momentos presenciales en el aula y el trabajo independiente que debe cumplir el estudiante, las estrategias y forma de valoración todo dentro de un entorno de la competencia profesional a que se aspira.

Para el desarrollo del modelo es importante que el docente disponga y utilice estrategias (Tobón, 2007) que favorezcan: i) la enseñanza desde la sensibilización, la atención, la adquisición, la recuperación, la cooperación, la transferencia y la actuación con valores y ética; ii) el aprendizaje desde la selección, organización, comparación, repetición y meta cognición; iii) el trabajo en equipo desde la motivación, el fortalecimiento de las emociones y desarrollo de actitudes sociales; iv) el desarrollo tecnológico e investigativo a través del saber hacer en un contexto de conocimiento, ética y responsabilidad social y aplicación en la industria; v) la valoración desde la apropiación de métodos, técnicas e instrumentos con sentido autónomo y prospectivo para el desarrollo de su perfil profesional en el área de la ingeniería.

\section{Estrategia pedagógica}

La estrategia pedagógica principal e integradora utilizada es la de proyecto de aula, (Tobón, 2007; ITESM, 2000) que permite fomentar el desarrollo del proceso de investigación formativa en el aula y tiene como objetivo que el estudiante desarrolle sus propias capacidades y potencialidades, encontrándole sentido a lo que aprende, hace y valora, todo lo anterior en concordancia con la educación del ingeniero del siglo 2020 (National Academy of Engineering, 2005).

\section{Metodología de desarrollo}

Los pasos seguidos para el desarrollo del proyecto son: i) el proyecto se inicia evidenciando una situación problemática por parte del docente, ii) se forman equipos de trabajo iii) se establece la metodología y estrategias pedagógicas complementarias que permitan encontrar alternativas de solución al problema. Todos los trabajos deben ser entregados al docente en medios magnéticos a través de la red virtual dispuesta por la universidad para el acompañamiento y seguimiento del proceso pedagógico e investigativo, además del acompañamiento que el docente realiza por medio de tutorías presénciales, iv) se define el sistema de evaluación, la cual siempre será participativa y tomará como base de participación la autoevaluación y co evaluación tanto individual al interior del grupo como genérica entre grupos, v) se organiza y distribuye las acciones que faciliten el desarrollo eficaz de las soluciones planteadas al problema, vi) se facilita al estudiante bibliografía especializada referente a tecnologías de soldadura, códigos y procedimientos de soldadura, además se propone para fines complementarios dentro del marco investigativo el uso de la base de datos proquest, ebrary y los recursos bibliográficos de la universidad.

Con el desarrollo del proyecto de aula los estudiantes se integran y participan en forma individual y por equipos, con roles y responsabilidades bien definidos, además se tiene contacto permanente con la industria, los estudiantes hacen visitas empresariales y resuelven dudas que surgen a medida se va desarrollando el trabajo encontrándole significado a lo que aprende y hace, validando el trabajo desarrollado en la misma empresa y con el acompañamiento de supervisores y trabajadores expertos en el tema, permitiéndole aún más potencializar las habilidades y conocimientos adquiridos en clase y a través de consultas bibliográficas especificas de alta calidad. También esta estrategia permite el manejo de aspectos formales para la redacción de artículos, monografía, ensayos, protocolos e informes técnicos afianzando el manejo de normas, estructuración de documentos, planteamientos y solución de problemas, manejo de tiempos y organización de actividades, etc.

\section{Guía de proyecto de aula}

La guía del proyecto de aula (ver tabla 1) presentada por el docente a los estudiante de quinto semestre de los programas de ingeniería industrial y mecánica de la Universidad Autónoma del Caribe en la asignatura laboratorio de procesos industriales I, da libertad de seleccionar la empresa 
con la cual va a desarrollar su propuesta para la elaboración y calificación de procedimientos bajo norma, permite que el estudiante decida si su procedimiento de soldadura a elaborar es para producción, recubrimiento superficial o reparación, permite que el estudiante decida que código aplicar acorde a la estructura que tome como referencia, normalmente el código seleccionado se ajusta a los requerimientos del producto soldado que fabrica, etc.

Una vez desarrollado el proyecto se debe verificar que el informe o artículo preferentemente se ajuste a las consideraciones para publicaciones en revistas indexadas (Valderrama, 2005) puesto que permite mostrar de manera lógica y estructurada los resultados del trabajo desarrollado preparando al estudiante para presentación de trabajos fruto de investigación en sentido estricto (Restrepo, 2002) y el fortalecimiento de semilleros de investigación. El informe del proyecto de aula debe contener entre otros ítems la síntesis conceptual sobre experiencias obtenidas en cada una de las actividades requeridas y resumen de competencias adquiridas con el trabajo, resultados e interpretación de resultados obtenidos en inspección visual y ensayos aplicados, documentos del procedimiento calificado, análisis y relación de cálculo y costos de soldadura, conclusiones.

Tabla 1: Guía de Proyecto de Aula

\begin{tabular}{|c|c|}
\hline \multicolumn{2}{|r|}{ Objetivos } \\
\hline 1 & $\begin{array}{l}\text { Desarrollar la capacidad para interpretar, describir y aplicar los conceptos inherentes a la } \\
\text { tecnología de soldadura. }\end{array}$ \\
\hline 2 & $\begin{array}{l}\text { Desarrollar la competencia de emitir conceptos de forma crítica mediante la realización de } \\
\text { análisis cualitativos y cuantitativos soportados técnicamente. }\end{array}$ \\
\hline 3 & $\begin{array}{l}\text { Inspeccionar visual y por medio de ensayos las uniones soldadas acorde al código aplicable } \\
\text { identificando las discontinuidades y defectos presentes en las mismas. }\end{array}$ \\
\hline 4 & $\begin{array}{l}\text { Elaborar y calificar procedimientos de soldadura y habilidad del soldador acorde a códigos y } \\
\text { normas vigentes para la fabricación de productos metálicos soldados. }\end{array}$ \\
\hline 5 & Trabajar en equipo para el cumplimiento de las metas propuestas. \\
\hline 6 & Desarrollar la habilidad en la elaboración de artículos e informes técnicos. \\
\hline \multicolumn{2}{|r|}{ Conceptos relacionados para el desarrollo del trabajo } \\
\hline 1 & Tecnología de soldadura y seguridad en la fabricación de productos soldados. \\
\hline 2 & $\begin{array}{l}\text { Introducción a los códigos A.S.M.E. Tanque y recipientes a presión - Sección IX, A.P.I. 1104, } \\
\text { A.W.S. D1.1. }\end{array}$ \\
\hline 3 & Discontinuidades y defectos, criterios de aceptación y rechazo según códigos. \\
\hline 4 & $\begin{array}{l}\text { Métodos de inspección y pruebas (ensayos de tensión, doblez, dureza, sanidad, pruebas } \\
\text { hidrostáticas, inspección visual, tintas penetrantes, radiografías, ultrasonido, partículas } \\
\text { magnéticas, macroataque y microataque) }\end{array}$ \\
\hline 5 & $\begin{array}{l}\text { Procedimientos de soldadura y calificación de soldadores para el control y aseguramiento de } \\
\text { la unión soldada. }\end{array}$ \\
\hline \multicolumn{2}{|r|}{ Fecha de entrega y resultados (segundo corte) } \\
\hline 1 & Especificaciones iniciales del procedimiento de soldadura (E.P.S. preliminar). - Semana 1. \\
\hline 2 & Probeta para soldadura según diseño de junta especificado en E.P.S. preliminar. Semana 2. \\
\hline 3 & Unión soldada y aprobada por inspección visual según código especificado. Semana 3. \\
\hline 4 & Cupones de prueba ensayados acorde con código especificado. Semana 4. \\
\hline 5 & Paper y sustentación del paper: Semana 5. \\
\hline \multicolumn{2}{|c|}{ Integrantes por grupo } \\
\hline \multicolumn{2}{|c|}{ Entre tres (3) y cinco (5) personas } \\
\hline \multicolumn{2}{|r|}{ Observaciones } \\
\hline \multicolumn{2}{|r|}{$\begin{array}{l}\text { Todos los resultados deberán estar asociados con empresas del sector metalmecánico y ser } \\
\text { socializados en el aula de clase. Todos los equipos cuentan con las tutorías del docente en el } \\
\text { horario asignado para el mismo }\end{array}$} \\
\hline
\end{tabular}

El docente debe acompañar la guía para la formulación del proyecto de aula explicando como se va a desarrollar los conceptos y conocimiento necesarios para su ejecución e indicando como va a ser la valoración, acompañamiento y seguimiento de los resultados obtenidos en cada fase del proyecto. 


\section{METODOLOGÍA PARA LA ELABORACIÓN DEL PROCEDIMIENTO DE SOLDADURA}

Esta metodología para la elaboración y calificación del procedimiento de soldadura es recomendado a industrias que se dedican a: i) la fabricación de estructuras para edificios, puentes y otras estructuras metálicas, ii) las industrias relacionadas con la fabricación y montaje de plantas térmicas, tanques y recipientes a presión, tuberías y otros equipos y líneas de distribución trabajando a presión, iii) las empresas dedicadas a la fabricación y montaje de equipos y tuberías para el transporte y almacenamiento de hidrocarburos, iv) la fabricación de equipos de transporte tales como: aeronaves, automóviles, barcos, trenes y líneas férreas, equipos para carreteras, etc.,v) la fabricación de equipos y aparatos de consumo domestico, la cual frecuentemente necesita el cumplimiento de requisitos específicos de calidad en las soldaduras.

La secuencia general para la elaboración y calificación del procedimiento de soldadura es relacionada a continuación acorde con los siguientes lineamientos:

\section{Reconocimiento del proyecto o producto soldado a fabricar}

En este punto es importante la recolección de información necesaria para el desarrollo del procedimiento ya que permite identificar que se va a producir, que material se empleará, rango de espesores del material a soldar, tipos de juntas presentes en la construcción soldada y todas aquellas características y especificaciones indispensables para el producto con respecto a la unión soldada.

\section{Selección del código a trabajar}

Para la elaboración del procedimiento de soldadura es necesario determinar que tipo de construcción soldada se va a trabajar, para posteriormente seleccionar e implementar el código que más se adecue según los requerimientos.

\section{Definición de variables}

Es vital la definición de variables que permitan asegurar la compatibilidad entre material base y deposito de soldadura asegurando la calidad del producto acorde a las especificaciones de diseño y ejecución de la soldadura, entre las variables de soldadura se pueden identificar: las esenciales, esenciales suplementarias y las no esenciales.

\section{Elaboración del documento Especificación del Procedimiento de Soldadura - EPS preliminar}

Es la fase donde se listan las variables y parámetros de soldadura preliminares que aplican al producto soldado para que el soldador las ejecute sobre una probeta preparada para su soldadura y luego ser probadas determinando si las propiedades mecánicas de la unión soldada cumplen con lo especificado en el diseño. La EPS preliminar puede ser diseñada de acuerdo con QW - 482 del código ASME - Tanques y recipientes a presión Sección IX, 2004 (ASME, 2004)

\section{Preparación de la junta}

Es de vital importancia, preparar la junta (Niebles et al, 2008) antes de iniciar cualquier soldadura, por tanto se debe: (a) Identificar los parámetros de diseño de la junta. Aquí el código especifica la forma y geometría de los especimenes de prueba (probeta a soldar) una vez este identificado el tipo de junta aplicable. (b) Corte de los especimenes de prueba. Después de identificar el material, y analizar sus características, se debe trazar el material acorde a las dimensiones que va a poseer la probeta, para así proseguir al corte que puede ser mecánico o térmico. (c) Preparación y limpieza de los bordes. Es clave en el procedimiento de la soldadura, preparar los bordes de la pieza acorde al diseño y tipo de junta, además se debe retirar o remover impurezas o contaminantes y todo aquello que impida que la soldadura sea efectiva. (d) Ensamble de la junta. Para el ensamble de juntas a tope se debe tener en cuenta que ésta debe estar alineada para que la unión sea uniforme, por lo que se recomienda la utilización de grapas cuando se desea soldar tubos y platinas, si es de filete se recomienda que los bordes estén rectos y no existan espaciamiento entre las superficies de contacto. 


\section{Desarrollo de la soldadura}

Se procede a soldar la junta bajo los procesos y parámetros de soldadura especificados en la EPS preliminar, en su ejecución es recomendado seleccionar al mejor soldador de la empresa, dado que ellos tienen confiabilidad y conocen del proceso y la técnica de soldadura que mejor aplica al diseño de la junta y pueden aportar ideas y conceptos valiosos y necesarios.

\section{Inspección visual}

La inspección visual (ANSI/AWS, 2000) es un método no destructivo de valioso control del proceso mediante el cual una soldadura desde su fase de preparación hasta su puesta en servicio pueden evaluarse de acuerdo con unos requerimientos aplicables con miras de asegurar la calidad de la soldadura, esta evaluación puede ser llevada a cabo por: en primera instancia por soldadores, supervisores de soldadura, inspectores de soldadura por parte de los contratistas, inspectores de soldadura por parte de los propietarios, o inspectores reguladores.

La inspección visual es desarrollada antes, durante y después de la soldadura y tiene en cuenta aspectos como: i) conformidad con el procedimiento de soldadura especificado para la unión soldada, ii) control en la preparación de la junta a soldar, iii) verificación de condiciones operativas de: equipos de soldadura (calibrados y en buen estado), materiales de aporte y consumibles, gases, materiales base, iv) calidad del cordón de soldadura de raíz, v) preparación de la raíz de la junta antes de soldar por el otro lado, vi) temperatura de precalentamiento y entre pasadas, vii) secuencia de pases de soldadura, viii) calidad de los cordones de relleno y presentación de la soldadura, ix) limpieza entre pasadas, x) apariencia, dimensionamiento final de la soldadura, xi) distorsión y deformación generada en la soldadura, xii) tratamiento post soldadura, xiii) otros detalles.

\section{Selección y aplicación de ensayos requeridos}

Para la selección de los ensayos requeridos es necesario: (a) Identificar los ensayos aplicables según código. Una vez soldada la junta se procede a seleccionar los ensayos acordes al tipo de junta, ya sea junta de filete o a tope para las platinas o junta de ranura o a tope para las tuberías tal como lo especifica el código seleccionado. Estas pruebas son diseñadas para evaluar el efecto de las técnicas de soldadura y la compatibilidad de los metales base y metales de aporte, algunas de las pruebas comúnmente utilizadas para la calificación de procedimientos son: Tensión, doblez transversal (de cara, raíz y lado) y longitudinal, saneidad, macroataque, rotura en filetes. (b) Elaboración y extracción de cupones de prueba. Las dimensiones necesarias de los cupones de prueba están reglamentadas por el código estipulando la forma final de la unión soldada para así evaluar la efectividad de la soldadura. (c) Aplicación de los ensayos. Estos deben ser aplicados una vez sea aprobada la unión soldada por inspección visual y los criterios de aceptación y de aplicación están definidos por los códigos, para su aplicación se requiere personal con entrenamiento y certificación en cada ensayo aplicable.

\section{Registro de calificación del procedimiento RCP}

Una vez aplicados los ensayos y determinado que las pruebas cumplen con las especificaciones para lo cual fue concebido en el diseño se procede a formalizar el procedimiento de soldadura registrando en un documento de registro de calificación de procedimiento de soldadura RCP las pruebas aplicadas, recuerde las calificaciones de procedimiento de soldadura se hacen para demostrar la compatibilidad existente entre los metales base, los materiales de aporte, los proceso de soldadura y la técnica aplicada, y el cual puede ser diseñado de acuerdo con QW - 483 del código ASME - Tanques y recipientes a presión Sección IX, 2004 (ASME, 2004)

\section{Edición del procedimiento de soldadura}

En esta fase se procede a editar el procedimiento de soldadura que será enviado a producción para la realización de las soldaduras que están cubiertas dentro de los rangos establecidos en el procedimiento de soldadura. 


\section{CALIFICACIÓN DE SOLDADORES Y OPERARIOS DE SOLDADURA}

Una vez que el procedimiento de soldadura ha sido calificado, no se puede utilizar hasta que los soldadores y operarios de soldadura hayan sido calificados de acuerdo con las especificaciones de ese procedimiento, por tanto las empresas que se desempeñen en el área de soldadura debe tener personal técnico altamente calificado que permita asegurar que el deposito de soldadura aplicado por el soldador esta acorde a las especificaciones del procedimiento de soldadura y que estos tienen la capacidad y habilidad para ejecutar satisfactoriamente las soldaduras de producción acorde a los criterios de aceptación previamente definidos en los códigos (ACS, 2003; Cary, 1992).

Para la calificación de soldadores y operarios de soldadura la metodología a seguir es muy similar a la de elaboración y calificación de procedimientos de soldadura, teniendo en cuenta que cuando se califica un procedimiento de soldadura se tiene como eje de acción demostrar la compatibilidad existente los metales base, los materiales de aporte, los proceso de soldadura y la técnica aplicada mientras que cuando se califica un soldador u operario de soldadura el eje de acción se centra en demostrar que este tiene la capacidad y habilidad para ejecutar una soldadura en determinada posición y acorde a unas especificaciones dadas en el procedimiento de soldadura estipulado para la aplicación requerida. Para la calificación de soldadores y operarios de soldadura es fundamental la definición de variables que permitan cumplir con lo especificado en el procedimiento entre las cuales se encuentran: la posición de soldadura, la configuración de la junta, el tipo y tamaño del electrodo, el proceso de soldadura, el tipo de metal base, el espesor del metal base y la técnica específica de soldadura.

La secuencia general para la calificación del soldador es: i) identificar las necesidades de calificación de soldador en cuanto a: Proceso o procesos de soldadura, posiciones de soldadura, materiales base (platina o tubería o mixta), rango de espesores y diámetros calificados, tipos de juntas (ranura o de filete), etc., ii) identificar e interpretar el procedimiento de soldadura especificado para la calificación. lii) identificar las variables esenciales, iv) verificar que el equipo de soldadura, los materiales base y de aporte sean los requeridos y estén en condiciones óptimas de trabajo (calibrados), v) verificar que la probeta de prueba cumpla con los requerimientos dimensionales y de forma especificados en el código para la calificación del soldador, vi) inspeccionar el desarrollo de la soldadura para asegurar que cumple con el procedimiento de soldadura especificado para la calificación, vii) seleccionar y aplicar los ensayos requeridos para evaluar las probetas soldadas las cuales pueden ser seleccionados acorde a los requerimientos de alta calidad del producto soldado y a los ensayos aplicables, pruebas destructivas o no destructivas, teniendo en cuenta especificaciones de los códigos, ventajas y limitaciones del método de inspección, estándares de aceptación, y costo, viii) elaborar el documento de calificación de la habilidad del soldador, el cual puede ser diseñado de acuerdo con QW - 484 A (ASME, 2004)

\section{CASO DE APLICACIÓN}

Como caso de aplicación se presenta uno de los trabajos desarrollado como proyecto de aula en la asignatura de Laboratorio de Procesos Industriales I. "Elaboración y calificación de procedimientos de soldadura bajo código ASME Tanques y recipientes a presión - Sección IX, 2004", cumpliendo con la metodología especificada en este artículo.

\section{Elaboración y calificación de procedimientos de soldadura}

El procedimiento de soldadura se desarrolla en la empresa COMDISTRAL para la soldadura de un "recipiente a presión" con conexiones en tubería de cuatro (4) y seis (6) pulgadas de diámetro, Schedule 40 y soldadura circunferenciales de cuerpo a cabeza del tanque en diámetro de dieciséis 16 pulgadas, el cual será soldado con proceso SMAW y con aplicación de junta a tope con preparación de bordes en $\mathrm{V}$ y es elaborado y calificado para cumplir con lo especificado en QW 201, responsabilidades del fabricante o contratista.

Con base en las especificaciones del producto se determina que el código aplicable es el código ASME -Tanques y recipientes a presión (ASME, 2004), lo que determina que la sección del código 
aplicable es la sección IX "Estándar para calificar procedimientos de soldadura y soldadura fuerte, soldadores, braceadores, y operarios de soldadura y soldadura fuerte", de los planos de fabricación se determinan las variables que se tomarán como referencia para ajustar los parámetros iniciales en la EPS preliminar; tales variables son: proceso de soldadura, diseño de la junta, material base, y con esta información se ajustan las demás variables a saber: características eléctricas, material de aporte posición del soldador, temperatura de precalentamiento, técnica de soldadura, etc.

\section{Preparación de la junta}

Se tiene en cuenta el diseño de la junta, determinando la longitud de los tubos para la preparación de las probetas teniendo en cuanta los ensayos que aplican y las dimensiones de los cupones de prueba, especificado en la EPS preliminar y toma como referencia una junta a tope, bisel en $\mathrm{V}$ en una tubería de 6" de diámetro y cuyo espesor es de $0.280 "$. El corte del tubo se practicó con sierra eléctrica teniendo en cuenta las dimensiones especificadas para posteriormente realizar los biseles por medio de mecanizado con torno. Para el ensamble de la junta se hizo necesario la limpieza superficial de la pieza a una pulgada del borde tanto interna como externamente y de igual forma la alineación de la pieza permitiendo unir las dos partes que la conforman, por medio de grapas, las cuales tenían una separación de $120^{\circ}$ una con respecto a la otra.

\section{Desarrollo de la soldadura.}

Para la soldadura se seleccionó a uno de los mejores soldadores de la empresa COMDISTRAL, empresa con certificación ASME y la cual apoyo en el proceso a los estudiantes para el desarrollo del proyecto, quien soldó la junta de acuerdo a lo especificado en la EPS preliminar, utilizando para tal fin el proceso de soldadura SMAW, y material de aporte E6010 para pase de raíz y E7018 para pases de relleno y presentación ambos de 1/8" de diámetro, la junta fue soldada en la posición 1G (QW 122.1 y QW 461.4 a), en la cual el eje del tubo esta en posición horizontal y gira durante la aplicación de la soldadura.

\section{Inspección visual}

La inspección visual fue realizada verificando que: las probetas estén acorde con el diseño para esto se observó la preparación de la junta QW - 402, su montaje, alineamiento y limpieza; los materiales base y de aporte QW - 403 / QW - 404 sean los especificados y mantenidos acorde con sus especificaciones; durante el proceso de la soldadura se observó que soldador cumpliera lo especificado en el procedimiento para cada pase aplicado sobre la junta, esto es con respecto a la limpieza entre los cordones, que se apliquen las temperaturas de precalentamiento QW - 406, que las características eléctricas QW 409 estén de conformidad con la EPS, que la técnica de soldadura aplicada sea la correcta QW - 410; después de realizado el proceso de soldadura se verificó que el tamaño de soldadura sea el adecuado, la apariencia final de la soldadura este conforme, así mismo si la soldadura cumple con los criterios de aceptación especificados en QW 144 y QW-194 .

\section{Selección y aplicación de ensayos requeridos.}

Para la selección de los ensayos que aplican acorde al espesor de metal base requerido para calificación se tuvo en cuenta el ítem QW-451 que hace referencia a "Límites de Espesores y Especimenes de Ensayos Para Calificación de Procedimientos de Soldadura" determinando que los ensayos a aplicar son: Dos (2) de doblez de cara, dos (2) de doblez de raíz, y dos (2) de tensión, para definir el orden de remoción de los cupones de prueba se tuvo en cuenta el ítem QW-463.1.d que aplica para tuberías y las dimensiones y criterios de aceptación de cada cupón de prueba ensayado son para: Prueba de tensión acorde con QW -151.2 y QW - 462.b, tiene como procedimiento de prueba QW - 152 y se acepta acorde con QW - 153.1; doblez de cara y raíz transversales acorde con QW - 462.3.a, tiene como procedimiento de prueba QW -162, se conduce acorde con QW - 466.1 y se acepta acorde con QW - 163. Al aplicar las pruebas en un laboratorio certificado, estas evidencian que cumplen con los criterios de aceptación establecidos en el código. 
Registro de calificación del procedimiento. RCP

Por último se elaboró el RCP (ver tabla 2) con base a lo estipulado en la EPS y los resultados obtenidos en los ensayos y cumpliendo con lo estipulado en QW 200.2, tomando en cuenta que la información contenida en el RCP es un registro real de las condiciones usadas para soldar la probeta.

Tabla 2: Formato del Registro de Calificación del Procedimiento de Soldadura

$$
\text { QW - } 483 \text { REGISTRO DE LA CALIFICACIÓN DEL PROCEDIMIENTO RCP }
$$

Nombre de la Compañía: INDUSTRIAL WELDING Registro de la Calificación del Procedimiento: 000 JUNTAS (QW - 402)

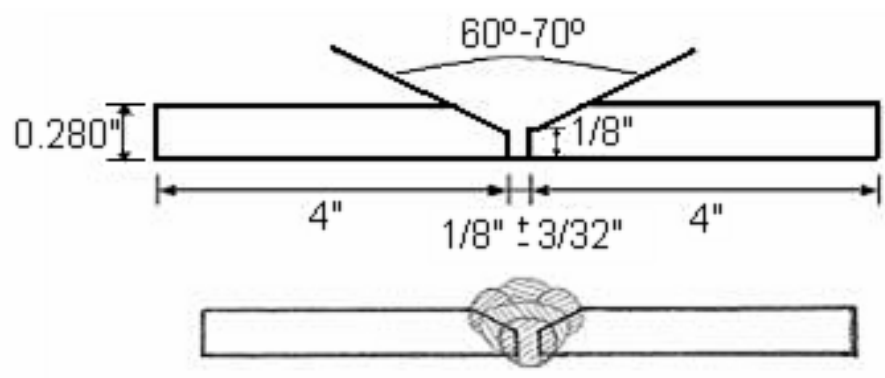

METALES BASE (QW - 403)

No. P1 Grupo No.1 a No. P1 Grupo No.1; Especificación, Tipo y Grado: SA 106 Gr B Tipo: Tubo ; Tamaño nominal: 6" Sch 40

CARACTERÍSTICAS ELÉCTRICAS (QW - 409)

Para electrodo E 6010

Corriente: continua Polaridad: inversa / positiva Intensidad: $60 \mathrm{~A}$ - 80A Tensión 18V - 24V

Para electrodo E 7018

Corriente: continua Polaridad: inversa / positiva

Intensidad: 100A - 120A Tensión 20V - 24V
METALES DE RELLENO (QW - 404)

Especificación No. SFA 5.1

Clase AWS No. E - 6010 y E 7018

Diámetro: 1/8"

PRECALENTAMIENTO (QW - 406)

Temperatura Precalentamiento: $90^{\circ}-150^{\circ}$

TÉCNICA (QW - 410)

Cordoneado y oscilación máxima de 2.5 veces el diámetro del electrodo

Limpieza Inicial y Entre Pasadas con cepillado manual y esmerilado.

Prueba de Tracción (QW - 150): Aceptable, falla dúctil en material base.

Pruebas de Doblez Guiado (QW - 160)

\begin{tabular}{|l|l|}
\hline MARCA & RESULTADO \\
\hline DC -1, DC -2 & Aceptables, sin defectos. \\
\hline DR -1, DC -2 & Aceptables, sin defectos. \\
\hline
\end{tabular}

Nombre del Soldador:

Pruebas Conducidas por: EENN

\section{Estampe No.}

Prueba de Laboratorio No.

Certificamos que los informes en este registro son correctos y que las soldaduras de prueba fueron preparadas, soldados, y ensayados de acuerdo con los requerimientos de la Sección IX del Código ASME - Tanques y recipientes a presión.

Aprobado por: Enrique Niebles $\mathrm{N}$. Fecha: -------------

Edición del procedimiento de soldadura.

Una vez calificado, aprobado y registrado el procedimiento (ver tabla 3) se procedió a su edición acorde con lo especificado en QW 100 - 3, QW 200.1. y QW 482, manifestando que es obligatorio su cumplimiento por cada una de las partes involucradas en su aplicación en la empresa. 
Tabla 3: Especificación del Procedimiento de Soldadura.

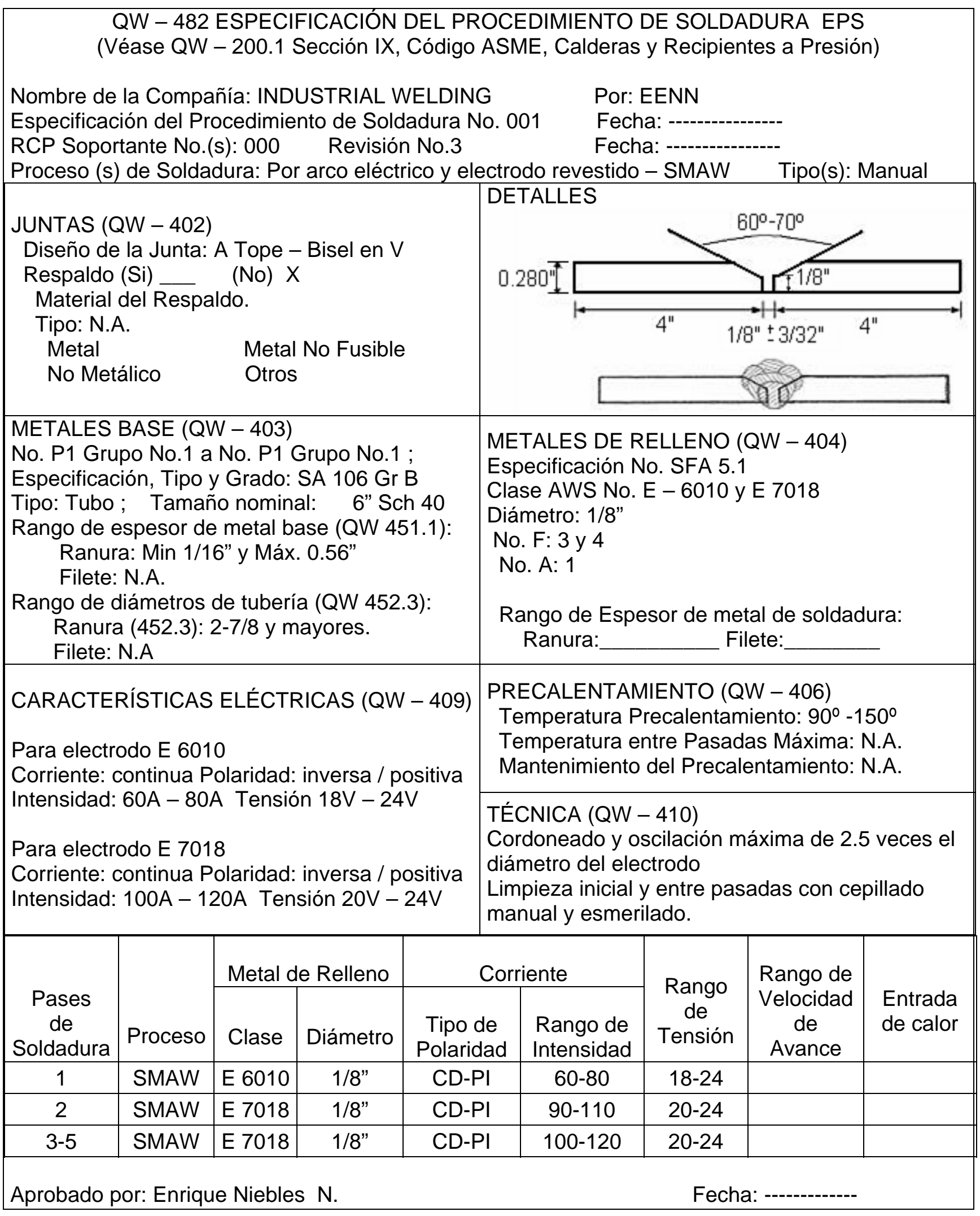

\section{RESULTADOS Y DISCUSIÓN}

Al inicio del proyecto no existía cultura en la realización de este tipo de proyectos y la metodología para su realización, lo que generaba resistencia en estudiantes y profesores del área por la responsabilidad y carga de trabajo que representa la formación por proyectos en el contexto de las competencias; por lo que fue necesario motivarlos a través de: i) introducir el desarrollo de este tipo 
de trabajos gradualmente e ir observando las bondades y resultados obtenidos ii) incentivar al mejor trabajo con su publicación en la revista de la facultad y participación en congresos estudiantiles, al igual que con proyectos en la feria de ciencia y tecnología de la universidad iii) invitar a empresarios amigos expertos del área a presentar ciclos de conferencias y de motivación a estudiantes semestralmente, iv) visitas empresariales, v) con un proceso sostenido de autoevaluación y co evaluación entre ellos mismos, con la asistencia y seguimiento del docente, que refleje los avances obtenidos en el semestre, es importante verificar que todos los miembros del equipo trabajen, sin embargo cuando un miembro no trabaja, lo cual no es muy común porque entre ellos mismos y el docente se regula el trabajo, se dialoga con él y se establecen compromisos. Una vez motivados y generado la cultura de trabajo con proyectos asociados a empresas los resultados obtenidos son los mejores, se han publicado artículos en la revista de la facultad, se ha participado con ponencias en congresos nacionales de estudiantes, se ha fortalecido la relación universidad-empresa, etc.

Con respecto a los empresarios, se encontró en algunos de ellos resistencia, por temor a divulgar la información de la empresa a terceros; ante esto, se les explica la confidencialidad de la información obtenida y los beneficios y necesidades de la relación universidad - empresa; por lo general las grandes empresas muestran gran voluntad de apoyo al proceso. Una vez aceptada la participación de los estudiantes en la empresa, estos la visitan y realizan el proyecto, se apoyan en expertos del área, entregan resultados y documentos obtenidos, manifiestan fortalezas o debilidades en las mismas con respecto al proyecto desarrollado y lo más importante los empresarios muestran interés de seguir apoyando a los estudiantes y docentes en el desarrollo de este tipo de trabajos.

\section{CONCLUSIONES}

En referencia a lo anteriormente expuesto, se concluye lo siguiente:

i) Al aplicar la propuesta de enseñanza el estudiante tiene contacto con la industria, está motivado, fortalece el saber-hacer y el saber-ser en concordancia con las competencias del ingeniero para el trabajo en campo de la industria, con el acompañamiento de supervisores y trabajadores expertos.

ii) El estudiante afianza el manejo de normas, estructuración de documentos, planteamientos y solución de problemas, manejo de tiempos y organización de actividades, despertando en ellos la motivación para pertenecer a semilleros de investigación, investigar en el área de tecnologías de soldadura y afines, participar en seminarios y congresos.

iii) La metodología empleada para la elaboración de procedimientos de soldadura y calificación del soldador bajo código se convierte en una herramienta de trabajo en el aseguramiento de calidad de la unión soldada que facilita la elaboración e interpretación de las especificaciones del procedimiento, el registro de la calificación del mismo y la calificación del soldador a supervisores, técnicos e ingenieros y personal afín con la tecnología de soldadura.

\section{REFERENCIAS}

A.C.S.; Asociación Colombiana de soldadura, Preparación para la calificación y certificación de inspectores de construcciones soldadas. Capítulos 4, 5, 8. Colombia (2003).

ANSI/AWS B1.11-2000. American Welding Society. Guide for the Visual Inspection of Welds (2000).

ASME - Boiler and pressure vessel, section IX - Qualification standard for welding and brazing procedure, welders, brazers, and welding and brazing operator. 13-190. USA. (2004).

Baldomir, L A., R Plaza, E. Isis y V. Ignoto; Evaluación de procedimientos de soldadura para revestimientos duros aplicados por el Proceso FCAW con transferencia metálica pulsada. Rev. Facultad de Ingeniería Universidad Central de Venezuela. ISSN 0798-4065 (en línea). 20(4), p.35-41. Octubre de 2005. http://www2.scielo.org.ve/scielo.php?script=sci arttext\&pid=S079840652005000400003\&lng=es\&nrm=iso. Acceso: 23 de Julio (2008). 
Bolaños, C.C., J.S. Rivas y J.J. Coronado; Caracterización de las propiedades mecánicas y microestructurales de soldadura empleadas en la recuperación de ejes, Facultad de Ingeniería Universidad de Antioquia: No 36, 33-43 (2006).

Camilleri D., P Molliconi y T. Gray; Computational Methods and Experimental Validation of Welding Distortion Models, Journal of Materials: Design and Applications: 221(L4), 235-249 (2007).

Cary, H.B.; Manual de Soldadura Moderna, $2^{\mathrm{a}}$ edición, tomo 3, 671-691, editorial Prentice-hall, México (1992).

Dunder M.; Cost Effective Automation of Pipe to Pipe Chamber Welding Process in Steamboilers Production, $10^{\text {th }}$ International Research/Expert Conference "Trends in the Development of Machinery and Associated Technology" TMT 2006, 441-444 Barcelona, Spain 11 to 15 September (2006).

ITESM, Instituto Tecnológico de Monterrey; Las técnicas didácticas en el modelo educativo del Tec de Monterrey. (2000) http://www.sistema.itesm.mx/va/dide/documentos/inf-doc/tecnicas-modelo.PDF. Acceso: 21 de julio (2008).

Méndez P. F.; Investigación en Soldadura en el MIT y Panorama de la Industria de la Soldadura en los Estados Unidos, Presentación principal de la conferencia "Tecnologías de la Soldadura y Unión de Materiales", San Sebastián, España 1 y 2 de Diciembre (1999). Acceso: 23 de Julio (2008).http://www.mines.edu/ pmendez/Publications/Papers/1999spain.pdf

National Academy of Engineering, (2005). Educating the Engineer of 2020. 71-73 http://www.nap.edu/catalog/11338.html\#toc, (2007).

Niebles, E.; Modelo de diseño y base de conocimiento en tecnologías de soldadura para el desarrollo de productos soldados, Cienthia y Technica, ISSN 0122-1701; (en línea), 37 (1), 473-478. (2007). http://www.utp.edu.co/ciencia/index.php?UnArt=32\&id=942\&anoFecha=2007\&mesFecha=9

Niebles E., H. Maury y J. Torres; Diseño para la fabricación y ensamble de productos soldados: Un enfoque metodológico y tecnológico, $1^{\mathrm{a}}$ edición, Ediciones Uninorte, Barranquilla-Colombia (2008).

Restrepo B.; Conceptos y aplicaciones de la investigación formativa y criterios para evaluar la investigación científica en sentido estricto, 3-14. (2002). http://www.cna.gov.co/cont/ doc_aca/index.htm. (2007).

SENA - Servicio Nacional de aprendizaje.; Caracterización Ocupacional Área de Soldadura. 107109, Bogotá, Colombia (2006). www.sena.edu.co

Tobón, S.; Formación basada en competencias, 2ª edición, 129-162. Ecoe ediciones. Bogotá (2007).

Valderrama, J.O.; Principales Aspectos sobre la preparación de un artículo para ser publicado en una revista internacional de corriente principal, Rev. Información Tecnológica, ISSN 0718-0764; (en línea): 16(2), 3-14. (2005). http://www.scielo.cl/scielo.php?script=sci arttext\&pid=S0718$07642005000200002 \& \operatorname{lng}=$ es\&nrm=iso $(2008)$.

Wang T. M. y F. Liu; La Situación Actual y las Tendencias de Desarrollo en la Educación de la Ciencia de Materiales e Ingeniería en China, Rev. Journal of Materials Education: ISSN 0738-7989; (en línea): 26 (1-2), p 127-132. 2004. http://redalyc.uaemex.mx/redalyc/pdf/266/26626219.pdf. Acceso: 24 de julio (2008). 